\title{
Adapting efficiency of salt-sucrose mixtures
}

\author{
HARRY LAWLESS \\ Monell Chemical Senses Center, Philadelphia, Pennsylvania
}

\begin{abstract}
Previous studies had observed that $\mathrm{NaCl}$-sucrose mixtures cross-adapted solutions of their unmixed components just as effectively as adaptation to the components themselves, while quinine-sucrose mixtures were not as effective in cross-adapting their components. The present study investigated whether these effects were due to the different tastants involved or to different conditions of stimulation and adaptation employed. Adaptation to NaCl-sucrose mixtures using the conditions of the quinine-sucrose study resulted in perfect cross-adaptation of $\mathrm{NaCl}$ saltiness by the mixture, but only partial adaptation of sucrose sweetness by the mixture. A second study attempted to reconcile this remaining difference by examining the stimulation parameters of flow rate, temperature, and area stimulated. As flow rate and area were decreased, the difference between mixture adaptation and self-adaptation of sucrose was attenuated. Mixture suppression may depend upon the tastants mixed and the parameters of stimulation, and may be the result of several physiological mechanisms.
\end{abstract}

Two important perceptual characteristics of the sense of taste are the effects called adaptation and mixture suppression. When solutions of constant concentration flow over the dorsal anterior tongue surface, the perceived taste intensity decreases over time (McBurney \& Pfaffmann, 1963; Meiselman, 1975). This perceptual adaptation has a close correlate in recordings from primary afferent nerves (Diamant, Oakley, Strom, Wells, \& Zotterman, 1965; Pfaffmann, 1955). The perceived intensity of a taste substance is usually less when it is presented in a mixture with a tastant of another quality than when it is presented in an equimolar unmixed solution (Bartoshuk, 1975; Lawless, 1977). This effect is commonly called mixture suppression.

Recent research has addressed the adapting efficiency of taste mixtures (Kroeze, 1978, 1979; Lawless, 1982). When a mixture flows over the tongue and one of the components of that mixture is then presented, will the perceived intensity of the component be reduced to the same degree that it would have been if the adapting rinse had been the component itself? Intuitively, one might expect the mixture to be less effective at adapting the component solutions, since the perceived intensity of the components is suppressed in the mixture. However, recent perceptual experiments indicate that the mechanisms underlying suppression may be central to the site of gustatory adaptation (Bartoshuk, 1979; Lawless, 1979; Bartoshuk \& Seibyl, Note 1). If mech-

The author thanks Marc D. Corina for the design and construction of the apparatus and assistance in the execution of the experiment and analysis of the data. The author's mailing address is: Monell Chemical Senses Center, 3500 Market Street, Philadelphia, Pennsylvania 19104. anisms underlying gustatory adaptation are peripheral to the mechanisms giving rise to suppression, then the suppression should not affect the adapting efficiency of the mixture.

Previous studies of the adapting characteristics of mixtures have led to contradictory findings. Kroeze (1978, 1979) found that adaptation to salt-sucrose mixtures decreased the perceived intensity of subsequent salt and sucrose stimuli to the same extent as did self-adaptation to those stimuli. Lawless (1982), however, found that adaptation to quininesucrose mixtures left a residual sweet taste when followed by equimolar sucrose. This sweet taste was of greater magnitude than the taste left after adaptation to the unmixed sucrose itself. Similarly, a residual bitter taste was left when the mixture was followed by equimolar quinine. The efficiency of adaptation to salt and sucrose mixtures is compatible with a mechanism of suppression central to the site or sites of gustatory adaptation. The residual taste left after adaptation to quinine-sucrose mixtures, however, may imply at least one peripheral mechanism contributing to mixture suppression between sucrose and quinine.

These disparate findings could arise from several sources. First, mixture suppression between quinine and sucrose could be caused by a physiological mechanism different from that underlying suppression between sucrose and sodium chloride. That is, the difference may be due to the different tastants. Experiment 1 below addressed this possibility. Second, the difference may have arisen from the different conditions of stimulation in the two experiments. The studies of Lawless and Kroeze differed in temperature $\left(34^{\circ}\right.$ vs. $\left.21^{\circ} \mathrm{C}\right)$, flow rate $(4$ vs. $.8 \mathrm{ml} /$ $\mathrm{sec}$ ), and area of stimulation (anterior third of the tongue vs. a single anterior side of the tongue). Ex- 
periment 2 addressed the effects of these variables on the adapting efficiency of mixtures.

\section{EXPERIMENT 1}

This experiment attempted to replicate the effects of Kroeze $(1978,1979)$ for salt-sucrose mixtures, using the common dorsal-flow stimulation conditions employed by Lawless (1982) for sucrosequinine mixtures.

\section{Methods}

Subjeets. Ten healthy young adults were recruited from the staff of Monell and from the campus of the University of Pennsylvania. Nonstaff volunteers were paid SS to participate.

Stimull. Stimuli consisted of solutions of $.32 \mathrm{M}$ sucrose, $.32 \mathrm{M}$ $\mathrm{NaCl}$, and a mixture containing both $.32 \mathrm{M}$ sucrose and $.32 \mathrm{M}$ $\mathrm{NaCl}$ and deionized water. The mixture was not produced by physically pouring together the component solutions, which would have diluted them, but was prepared to be equimolar to the unmixed solutions. For example, the $.32-\mathrm{M}$ sucrose solution and the mixture both contained $109.8 \mathrm{~g}$ /liter of sucrose.

Procedures. Stimuli were flowed for $5-10 \mathrm{sec}$ over the dorsal anterior third of the tongue by means of a McBurney gravity flow system. All were preceded by a $30-\mathrm{sec}$ adapting rinse. Rinses and stimuli were presented at $4 \mathrm{ml} / \mathrm{sec}$ and $34^{\circ} \mathrm{C}$. Each subject was instructed to remain as still as possible during the flow, and to keep his/her lips closed around his/her extended tongue to prevent the flow from reaching unadapted areas. Switching from rinse to stimulus usually did not interrupt the flow.

The subjects were cued approximately $1 \mathrm{sec}$ before stimulus arrival. They were asked to judge peak taste intensity by the method of magnitude estimation without a modulus (Stevens, 1969). The subjects were instructed to assign numbers to stimuli in proportion to their perceived taste intensity, to assign these values to appropriate taste quality categories (sweet, salty, sour, bitter, or other), and to divide their total intensity judgments into two or more qualities if more than one quality was perceived.

Each stimulus also served as an adapting rinse. Exceptions were the combinations of water-after-water, water-after-mixture, and mixture-after-mixture, which were not considered germane to the questions of the present study. Each subject tasted the remaining 13 stimulus-rinse pairs twice in random orders.

Data analyols. Judgments for each session were multiplied by an individual constant chosen to make the grand mean for each subject and session equal to 200 . This preserved the proportions among judgments for each subject and brought each subject's judgments into the same range before averaging, in order to avoid undue weight in the judgments of subjects who used numbers with larger magnitudes.

\section{Results}

Figure 1 shows the mean sweetness and bitterness ratings for 11 of the 13 stimuli. The results for water-after-sucrose and water-after- $\mathrm{NaCl}$ are omitted because they did not produce sweet or salty tastes significantly different from zero. Paired comparisons were performed using both $t$ tests and Wilcoxon matched-pairs signed rank tests. The nonparametric tests were employed since some of the conditions were highly skewed. The following differences were significant by both tests at $p<.01$ unless otherwise stated.

Adaptation. The sweetness of sucrose was reduced $89 \%$ by a sucrose adapting rinse, as compared with

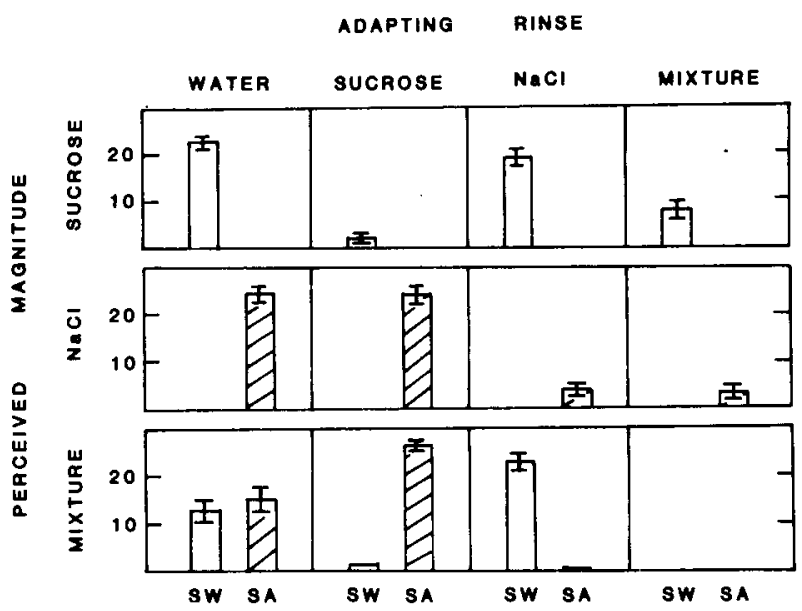

Figure 1. Mean aweetness (open bars) and saltiness (hatched bars) $\pm 1 \mathrm{SE}$ for $.32 \mathrm{M}$ sucrose, $.32 \mathrm{M} \mathrm{NaCl}$, and the mixture containing both $.32 \mathrm{M}$ sucrose and $32 \mathrm{M} \mathrm{NaCl}$ (rows) after the different adaptation conditions (columns).

the sweetness of sucrose after water. The saltiness of $\mathrm{NaCl}$ was reduced $83 \%$ by $\mathrm{NaCl}$ adaptation. Adaptation to the mixture reduced the saltiness of $\mathrm{NaCl}$ by $89 \%$ (not different from $\mathrm{NaCl}$ adaptation). In contrast to the results of Kroeze, adaptation to the mixture was not as effective as adaptation to sucrose in reducing the sweetness of sucrose, with a reduction of only $66 \%$ for mixture adaptation. That is, there was a reliable residual sweet taste after adaptation to the mixture (compare the second and fourth panels, top row, Figure 1). This residual sucrose sweetness was surprising, since $\mathrm{NaCl}$ reduced the sweetness of unmixed sucrose by $27 \%$, possibly due to the intrinsic sweet taste of $\mathrm{NaCl}$ (see Bartoshuk, Murphy, \& Cleveland, 1978).

Mixture suppression. The sweetness of the mixture after water was reduced by $43 \%$ as compared with the sweetness of equimolar unmixed sucrose after water. The saltiness of $\mathrm{NaCl}$ was reduced by $37 \%$ as compared with the saltiness of equimolar unmixed $\mathrm{NaCl}$ after water $(\mathrm{p}<.01, \mathrm{t}$ test, $\mathrm{p}<.05$, Wilcoxon).

Recovery from suppression. After adaptation to sucrose, the sweetness of the mixture was reduced by $94 \%$ and the saltiness recovered to the level at which it was perceived when unmixed, that is, not significantly different from the saltiness of $\mathrm{NaCl}$ after water. After adaptation to $\mathrm{NaCl}$, the saltiness of the mixture was reduced by $93 \%$ and the sweetness of the mixture returned to a level not different from that of unmixed sucrose after water.

\section{EXPERIMENT 2}

With the exception of the nonequivalence of mixture adaptation and self-adaptation of sucrose, Experiment 1 replicated the effects of mixture suppression and adaptation previously observed in salt- 
sucrose mixtures (Kroeze, 1978, 1979). Experiment 2 investigated three methodological variables potentially responsible for this discrepancy: flow rate, temperature, and area of stimulation.

\section{Methods}

Subjects. Ten healthy young persons were recruited from the campus of the University of Pennsylvania and were paid $\mathbf{\$ 1 0}$ each to participate.

Stimuli. Stimuli consisted of $.32 \mathrm{M}$ sucrose and the mixture of $.32 \mathrm{M}$ and $.32 \mathrm{M} \mathrm{NaCl}$ and deionized water. In all trials, unmixed sucrose was the test stimulus, while all three solutions were used as adapting rinses.

Procedure. Six conditions were run. Solutions flowed over the dorsal anterior third of the subject's extended tongue at $4 \mathrm{ml}$ / sec (bilateral stimulation), at $4 \mathrm{ml} / \mathrm{sec}$ over one side of the subject's tongue, or at $.8 \mathrm{ml} / \mathrm{sec}$ over one side. Unilateral restriction was achieved by means of a barrier constructed of parafilm stretched over a wire frame and held in place by the subject. In addition, each of these three conditions was run both at $34^{\circ} \mathrm{C}$ and at room temperature (approximately $21^{\circ} \mathrm{C}$ ). The condition of $34^{\circ}$, $4 \mathrm{ml} / \mathrm{sec}$, and bilateral stimulation was the same as in Lawless (1982) and Experiment 1, while the condition of $21^{\circ}, .8 \mathrm{ml} / \mathrm{sec}$, and restricted stimulation approximated the conditions used by Kroeze (1978). The fourth possible combination of flow rate and area, $.8 \mathrm{ml} / \mathrm{sec}$ and bilateral stimulation, was omitted due to difficulties in controlling the area over which the stimulus flowed.

The subjects served in two sessions, with the three conditions of (1) $4 \mathrm{ml} / \mathrm{sec}, 35^{\circ}$, bilateral stimulation, (2) $4 \mathrm{ml} / \mathrm{sec}, 35^{\circ}$, restricted stimulation, and (3) $.8 \mathrm{ml}, 21^{\circ}$, and restricted stimulation in one session, and the three remaining conditions in the other session. Each of the three stimulus-rinse pairs was tasted three times by each subject. Ratings were made by magnitude estimation, as in Experiment 1, and the same normalization procedure was performed.

\section{Results}

Normalized data were analyzed by means of a repeated-measures analysis of variance using condition (flow-rate/area combination), temperature, adapting rinse, and replicates as factors. Main effects of condition $[F(2,18)=5.80, \mathrm{p}<.05]$, adapting rinse $[F(2,18)=48.47, p<.001]$, and the adapting rinse $x$ condition interaction $[F(4,36)=3.92, p<.01]$ were significant.

Figure 2 shows the mean sweetness judgments for the three conditions and the three adapting rinses averaged across temperatures. The condition of $4 \mathrm{ml} / \mathrm{sec}$ and bilateral stimulation (no barrier) replicated the effects of Experiment 1. Adaptation to the mixture left a stronger residual sweet taste than did adaptation to sucrose $[\mathrm{t}(118)=2.64, \mathrm{p}<.05]$. Restricting the stimulated area to one side of the tongue also left a residual sweet taste greater than that from self-adaptation $[\mathrm{t}(118)=2.90, \mathrm{p}<.01]$, although the magnitude of the difference was slightly attenuated. Both restricting the area and reducing the flow rate (rightmost panel in Figure 2) replicated the effect observed by Kroeze (1978) of no difference between mixture adaptation and self-adaptation.

While lowering the temperature appeared to reduce the difference between mixture adaptation and self-adaptation (mean difference of 2.2 for $34^{\circ}$

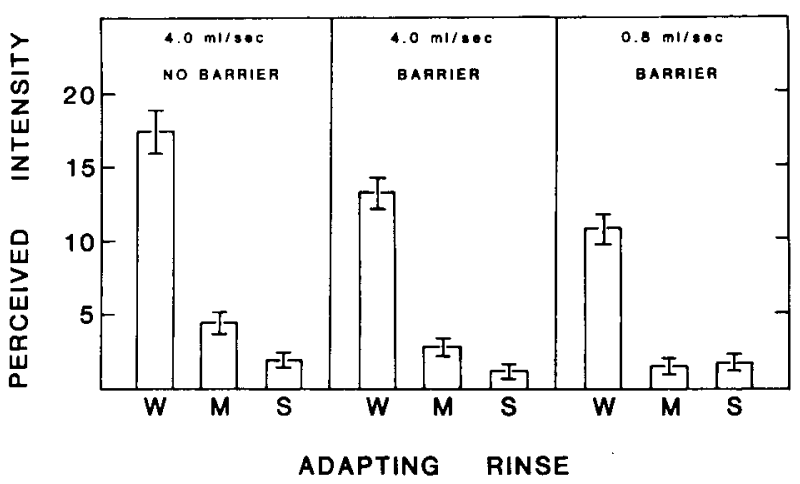

Figure 2. Mean sweetness ( $\pm 1 \mathrm{SE}$ ) for the conditions of $4.0 \mathrm{ml} /$ sec flow with bilateral stimulation, $4.0 \mathrm{ml} / \mathrm{sec}$ with restricted (unilateral) stimulation, and $.8 \mathrm{ml} / \mathrm{sec}$ with restricted stimulation, averaged across both temperatures.

and .3 for $22^{\circ}$, collapsed across conditions), neither the effect of temperature nor any interactions with temperature achieved statistical significance.

\section{Discussion}

When one component of a mixture is perceptually "removed" by adaptation, the other component returns to the intensity in the unmixed state. This release from mixture suppression appears to be a robust effect observed with many two-component mixtures (Bartoshuk, 1979; Lawless, 1979; Bartoshuk \& Seibyl, Note 1). This effect is generally interpreted as being consistent with mechanisms that underlie mixture suppression which are central to the site of mechanisms that underlie gustatory adaptation in the afferent pathway, since the presence of suppression appears to depend upon the presence of a neural signal for both components, regardless of the fact that a mixture is still chemically present on the tongue.

The converse of this principle is that a mixture should be just as effective as its unmixed components in adapting the tastes of those components. In other words, while mixture suppression is dependent upon the perceived intensity of components, adaptation should be dependent upon physical concentration alone. While this was observed by Kroeze for saltsucrose mixtures, nonequivalence in adapting efficiency was observed by Lawless for sucrose-quinine mixtures. The decreased adapting efficiency of mixtures suggests the possible existence of peripheral mechanisms for mixture suppression in addition to those central mechanisms implied by the release from suppression.

The existence of multiple physiological mechanisms that underlie suppression would not be inconsistent with current electrophysiological observations. Potentially suppressive interactions can be observed at several levels of the afferent pathway. Suppressive effects have been observed in response to mixtures in 
the hamster chorda tympani (Hyman \& Frank, $1980 \mathrm{a}, 1980 \mathrm{~b})$. In the geniculate ganglion of the cat, cells that are excited by amino acids that taste sweet to humans are inhibited by amino acids that taste bitter to humans (Boudreau, Oravec, \& White, 1981). At least one potential substrate for mixture suppression, then, lies at or before the primary afferent nerves. A more central mechanism for suppression is also possible. Nonadditive responses to mixtures have been observed in the nucleus of the solitary tract in the rat (Halpern, 1959). In the hamster, inhibition of quinine-sensitive units by sucrose stimulation has been observed in the nucleus of the solitary tract and the pontine taste area (Smith, Travers, \& Van Buskirk, 1979; Travers \& Smith, 1979).

The present experiment suggests some of the variables possibly responsible for the discrepancies between the findings with salt-sucrose mixtures and quinine-sucrose mixtures. The effects of size of area of stimulation and flow rate may each play a part in determining the equivalence of mixture adaptation and self-adaptation. Slower flow rates and decreased areas tend to decrease the intensity of taste solutions (Meiselman \& Bose, 1977; Smith, 1971) and also the adapting efficiency of mixtures. Whether this is a function of the decreased intensity per se is unclear.

The asymmetry of the decreased adapting efficiency of the mixture on sucrose but not on salt suggests the existence of different mechanisms for the suppression of saltiness by sucrose and of sweetness by $\mathrm{NaCl}$. Another asymmetry was noted by Bartoshuk (1979). The saltiness of $\mathrm{NaCl}$-quinine mixtures was released from suppression after adaptation to quinine, while the bitterness of the mixtures remained suppressed after adaptation to $\mathrm{NaCl}$. Bartoshuk interpreted this as implying a peripheral mechanism for the suppression of quinine bitterness by $\mathrm{NaCl}$. Thus, in addition to the conditions of stimulation, it appears that the nature of the individual chemicals or their particular taste qualities may also determine how mixture suppression occurs.

\section{REFERENCE NOTE}

1. Bartoshuk, L. M., \& Seibyl, J. P. Suppression of bitterness of $\mathrm{QHCl}$ in mixtures: Possible mechanisms. Paper presented at The Association for Chemoreception Sciences, Fourth Annual Meeting, Sarasota, Florida, April 18, 1982.

\section{RETERENCES}

Bartoshuk, L. M. Taste mixtures: Is mixture suppression related to compression? Physiology \& Behavior, 1975, 14, 643-649.

Bartoshuk, L. M. Taste interactions in mixtures of sucrose with $\mathrm{NaCl}$ and sucrose with $\mathrm{QHCl}$. Society of Neurosciences $A b$ stracts, 1979, 5, 125.

Bartoshuk, L. M., Murphy, C., \& Cleveland, C. T. Sweet taste of dilute $\mathrm{NaCl}$ : Psychophysical evidence for a sweet stimulus. Physiology \& Behavior, 1978, 21, 609-613.

Boudreau, J. C., Oravec, J., \& White, T. D. A peripheral neural correlate of the human fungiform sweet, bitter sensations. Chemical Senses, 1981, 6, 129-141.

Diamant, H., OAkley, B., Sthom, L., Wells, C., \& Zotterman, Y. A comparison of neural and psychophysical responses to taste stimuli in man. Acta Physiologica Scandinavica, 1965, 64, 67-74.

Halpern, B. P. Gustatory responses in the medulla oblongata of the rat. Unpublished dissertation, Brown University, 1959.

Hyman, A. M., \& Frank, M. E. Effects of binary taste stimuli on the neural activity of the hamster chorda tympani. Journal of General Physiology, 1980, 76, 125-142. (a)

Hyman, A. M., \& Frank, M. E. Sensitivities of single nerve fibers in the hamster chorda tympani to mixtures of taste stimuli. Journal of General Physiology, 1980, 76, 143-173. (b)

Kroeze, J. H. A. The taste of sodium chloride: Masking and adaptation. Chemical Senses and Flavour, 1978, 3, 443-449.

KROEZE, J. H. A. Masking and adaptation of sugar sweetness intensity. Physiology \& Behavior, 1979, 22, 347-351.

LAWLESS, H. T. The pleasantness of mixtures in taste and olfaction. Sensory Processes, 1977, 1, 227-237.

LAwLEs8, H. Evidence for neural inhibition in bittersweet taste mixtures. Journal of Comparative and Physiological Psychology, $1979,93,538-547$.

Lawless, H. Paradoxical adaptation to taste mixtures. Physiology \& Behavior, 1982, 25, 149-152.

McBurney, D. H., \& Pfaffmann, C. Gustatory adaptation to saliva and sodium chloride. Journal of Experimental Psychology, $1963,65,529-532$.

Meiselman, H. L. Effects of response task on taste adaptation. Perception \& Psychophysics, 1975, 17, 591-595.

Meiselman, H. L., \& Bose, H. E. Effects of flow rate on taste intensity ratings. Chemical Senses and Flavor, 1977, 2, 515-522.

Praffmann, C. Gustatory nerve impulses in rat, cat and rabbit. Journal of Neurophysiology, 1955, 18, 429-440.

SмIтн, D. V. Taste intensity as a function of area and concentration. Journal of Experimental Psychology, 1971, 87, 163-171.

Smith, D. V., Travers, J. B., \& Van Buskirk, R. L. Brainstem correlates of gustatory similarity in the hamster. Brain Research Bulletin, 1979, 4, 359.372.

Stevens, S. S. Sensory scales of taste intensity. Perception \& Psychophysics, 1969, 6, 302-308.

Travers, J. B., \& SmIth, D. V. Gustatory sensitivities in neurons of the hamster nucleus tractus solitarius. Sensory Processes, $1979,3,1-26$.

(Manuscript received May 17, 1982; accepted for publication July 10, 1982.) 